\title{
DIFFERENTIAL AMINO ACID UTILIZATION BY SPIROPLASMAS AND THE EFFECT ON GROWTH KINETICS
}

\author{
ALGEM PATTERSON, CLAUZELL STEVENS, REYNOLDS M. CODY,* \\ AND ROBERT T. GUDAUSKAS* \\ The George Washington Carver Agricultural Experiment Station Carver Research \\ Foundation, Tsukegee University, AL 36088, U.S.A. \\ *Department of Botany, Plant Pathology and Microbiology, Auburn, AL 36849, \\ U.S.A.
}

(Received November 21, 1985)

The amino acid requirements of Spiroplasma citri, S. floricola, S. melliform, SR-3, brevi and $S$. apis were determined in a chemically defined medium. Deletion of individual amino acids from an otherwise complete growth medium indicated that of the 20 amino acids tested, 14 were essential for good growth in S. citri, 10 in S. floricola, 10 in $S$. melliform, 10 in brevi, 7 in S. apis and 14 in SR-3. Asparagine, glutamic acid, glycine, leucine, proline, threonine and valine were essential for good growth for all spiroplasmas tested, but deletion of cysteine and serine had marginal or no significant effect on their growth. Requirements for other amino acids varied from nonessential to essential for growth. The specific growth rate of spiroplasmas was affected by the amino acid composition of the growth medium. For example, the specific growth rate of $S$. floricola in a medium deficient in threonine was $0.06 \times 10^{-2} \mathrm{hr}^{-1}$, however, the specific growth rate in medium containing $4.8 \times 10^{-2} \mathrm{M}$ amino acid was $4.2 \times 10^{-2} \mathrm{hr}^{-1}$.

Spiroplasmas are nutritionally fastidious microorganisms which, like mycoplasmas, need amino acids for good growth. When additional amounts of different combinations of amino acids and in some cases a supplement of a single amino acid, were added in chemically undefined media, growth was stimulated in some spiroplasmas (1-5). However, these earlier evaluations of amino acid utilization by spiroplasmas in chemically undefined media were made difficult by the composition of complex components, such as PPLO broth that contained a source of free amino acids. Growth studies of spiroplasmas in these media only showed whether amino acids were inhibitory or stimulatory when added. CHANG and CHEN (6) in their recent studies investigated amino acid requirements 
of two flower spiroplasmas (Spiroplasma floricola and SR-3 spiroplasma) and honeybee spiroplasma ( $S$. melliform) in a chemically defined medium. Amino acids were grouped into 10 combinations based on their structural differences and roles in biosynthesis of sugars. Both flower spiroplasmas grew in any of the amino acid combinations. However, only 4 amino acid groups supported growth of $S$. melliform.

The purpose of this investigation was to determine the differential amino acid requirements of $S$. citri, S. melliform and the flower spiroplasmas $S$. floricola, SR-3, brevi and $S$. apis using a chemically defined medium, and to determine the relationship between the specific growth rate of these spiroplasmas and the concentration of amino acids.

\section{MATERIALS AND METHODS}

Spiroplasma strains and growth of spiroplasmas. Six spiroplasma strains representing four serogroups were used in this study. S. citri (ATCC 27665) was obtained from the American Type Culture Collection (ATCC), Rockville, MD, 20852. S. floricola (strain 23-6, ATCC 9989), SR-3 spiroplasma (ATCC 3095), brevi spiroplasma (ATCC 33474) and $S$. melliform (strain AS 576, ATCC 29416) were obtained from R. E. Davis (USDA/AR, Beltsville, MD). S. apis (strain PPS1, ATCC 33450) was supplied by R. E. McCoy (University of Florida, Fort Lauderdale, FL).

Stock cultures of flower spiroplasmas and S. melliform were maintained and subcultured in the chemically defined medium (CDM) described by CHANG and CHEN (7). S. citri was grown in a modified version of CDM designated as CDMA. The lipid components dissolved per liter of CDMA were palmitic acid, $14 \mathrm{mg}$; oleic acid, $11 \mathrm{mg}$; cholesterol, $20 \mathrm{mg}$; $0.1 \mathrm{ml}$ of Tween 40 and 80 and $10 \mathrm{mg}$ each of phospholipids as follows: phosphatidylcholine dipalmitoyl, phosphatidylethanolamine, phosphatidylcholine dimyristoyl, and phosphatidylcholine dioleoyl (Sigma Chemical Company, St Louis, MO). The spiroplasmas were transferred for 8 to 26 passages before initiating growth studies. To prepare the inoculum for each pathogenic and flower spiroplasma, $0.1 \mathrm{ml}$ of $10^{-2}$ and $10^{-3}$ dilutions, respectively, of logarithmic phase cultures were transferred to 2-ml aliquots of medium and incubated at $30^{\circ} \mathrm{C}$. A chemically defined medium free of sugars and amino acids was used for the inoculum dilution.

Amino acid deletion. The deletion technique was used to determine the differential amino acid requirement. The concentration of twenty amino acids was the same as that in Chang and Chen's chemically defined medium (7). All amino acids were purchased from the United States Biochemical Corporation, Cleveland, $\mathrm{OH}$. Growth titer was determined by counting cells from three day old cultures of S. floricola, SR-3 and brevi; four day old cultures of S. apis; and eight day old cultures of $S$. melliform and $S$. citri. A haemocytometer was used 
with dark field microscope, as previously described (5). All growth determinations were replicated ten times. The growth yield of each amino acid was determined by subtracting the initial concentration of the spiroplasma from the final growth titer in the log growth phase. The percentage growth yield of spiroplasmas for each amino acid was expressed as a percentage of the growth yield of the control culture, which contained all 20 amino acids (complete control culture).

The effect of amino acid concentration on the specific growth rate. The specific growth rate relationship of spiroplasmas and the concentration of 20 different amino acids was determined as described by Stevens et al. (5). The specific growth rates were calculated as described elsewhere (8). The specific growth rate was calculated in the early phase of the logarithmic growth. The assessment of the specific growth rate was determined within two days for $S$. floricola and brevi,

Table 1. Effect of deletion of individual amino acids on growth yield of spiroplasmas grown in a chemically defined medium.

\begin{tabular}{|c|c|c|c|c|c|c|}
\hline \multirow{2}{*}{ Amino acids } & \multicolumn{6}{|c|}{ Growth yield titer $($ cells $/ \mathrm{ml})\left(\times 10^{3}\right)$} \\
\hline & SR-3 & S. floricola & brevi & S. apis & S. citri & S. melliform \\
\hline L-Alanine & $0.2(20)^{a}$ & $0.8(70)$ & $0.4(31)$ & $0.46(92)$ & $0.3(25)$ & $0.5(31)$ \\
\hline L-Arginine & $0.1(10)$ & $0.4(33)$ & $13(100)$ & $0.82(164)$ & $0.2(17)$ & $0.6(38)$ \\
\hline \multicolumn{7}{|l|}{ L-Aspartic } \\
\hline acid & $1.0(100)$ & $0.9(75)$ & $1.0(80)$ & $0.30(57)$ & $0.2(17)$ & $0.5(31)$ \\
\hline L-Asparagine & $-b(0)$ & $0.1(8)$ & $0.2(15)$ & $0.10(20)$ & $0.1(8)$ & $-(0)$ \\
\hline L-Cysteine & $1.0(100)$ & $1.2(100)$ & $1.0(80)$ & $0.40(80)$ & $1.1(92)$ & $1.4(90)$ \\
\hline \multicolumn{7}{|l|}{ L-Glutamic } \\
\hline acid & $-(0)$ & $0.1(8)$ & $0.1(8)$ & $0.03(7)$ & $0.2(17)$ & $0.2(12)$ \\
\hline L-Glutamine & $-(0)$ & $0.9(75)$ & $1.0(80)$ & $0.40(80)$ & $-(0)$ & $0.1(6)$ \\
\hline Glycine & $-(0)$ & $-(0)$ & $0.1(18)$ & $0.10(20)$ & $0.1(8)$ & $0.2(12)$ \\
\hline L-Histidine & $0.2(20)$ & $1.2(100)$ & $1.0(80)$ & $0.30(60)$ & $0.2(17)$ & $0.6(38)$ \\
\hline L-Isoleucine & $-(0)$ & $0.1(8)$ & $0.1(8)$ & $0.28(56)$ & - & $-(0)$ \\
\hline L-Leucine & $-(0)$ & $-(0)$ & $0.2(15)$ & $0.03(6)$ & $-(0)$ & $0.2(12)$ \\
\hline L-Lysine & $-(0)$ & $-(0)$ & $-(0)$ & $0.07(14)$ & $0.4(33)$ & $0.5(31)$ \\
\hline L-Methionine & $0.8(80)$ & $1.0(83)$ & $1.0(80)$ & $0.44(90)$ & $0.2(17)$ & $-(0)$ \\
\hline \multicolumn{7}{|l|}{ L-Phenyl- } \\
\hline alanine & $0.1(10)$ & $0.2(20)$ & $0.1(8)$ & 0.14 ( 28) & $0.1(8)$ & $0.5(31)$ \\
\hline L-Proline & $-(0)$ & $-(0)$ & $0.1(8)$ & $0.01(2)$ & $-(0)$ & $-(0)$ \\
\hline L-Serine & $0.9(90)$ & $1.1(92)$ & $1.0(80)$ & $0.40(80)$ & $1.0(83)$ & $1.2(75)$ \\
\hline L-Threonine & $-(0)$ & $-(0)$ & $-(0)$ & $0.01(2)$ & $-(0)$ & $-(0)$ \\
\hline L-Tryptophan & $0.3(31)$ & $0.8(70)$ & $1.0(80)$ & $0.34(70)$ & $0.4(33)$ & $0.5(31)$ \\
\hline L-Tyrosine & $0.9(90)$ & $1.2(100)$ & $1.1(85)$ & $0.54(108)$ & $0.3(25)$ & $0.7(44)$ \\
\hline L-Valine & $-(0)$ & $-(0)$ & $0.1(8)$ & $0.04(8)$ & $0.2(17)$ & 0.3 ( 19) \\
\hline \multicolumn{7}{|l|}{ Complete } \\
\hline $\begin{array}{l}\text { control } \\
\text { culture }\end{array}$ & $1.0(100)$ & $1.2(100)$ & $1.3(100)$ & $0.50(100)$ & $1.2(100)$ & $1.6(100)$ \\
\hline
\end{tabular}

a The yield of each amino acid expressed as a percentage $(\%)$ of the yield of the complete control culture is given in parenthesis.

$b$ means no growth yield. 

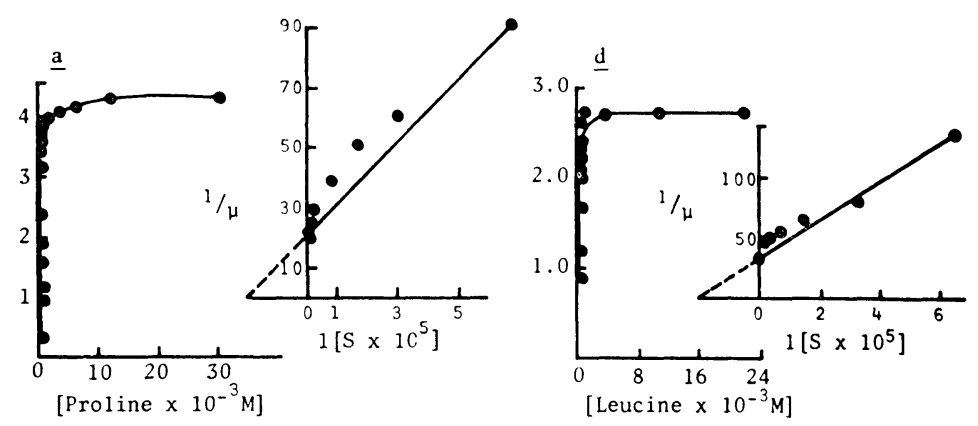


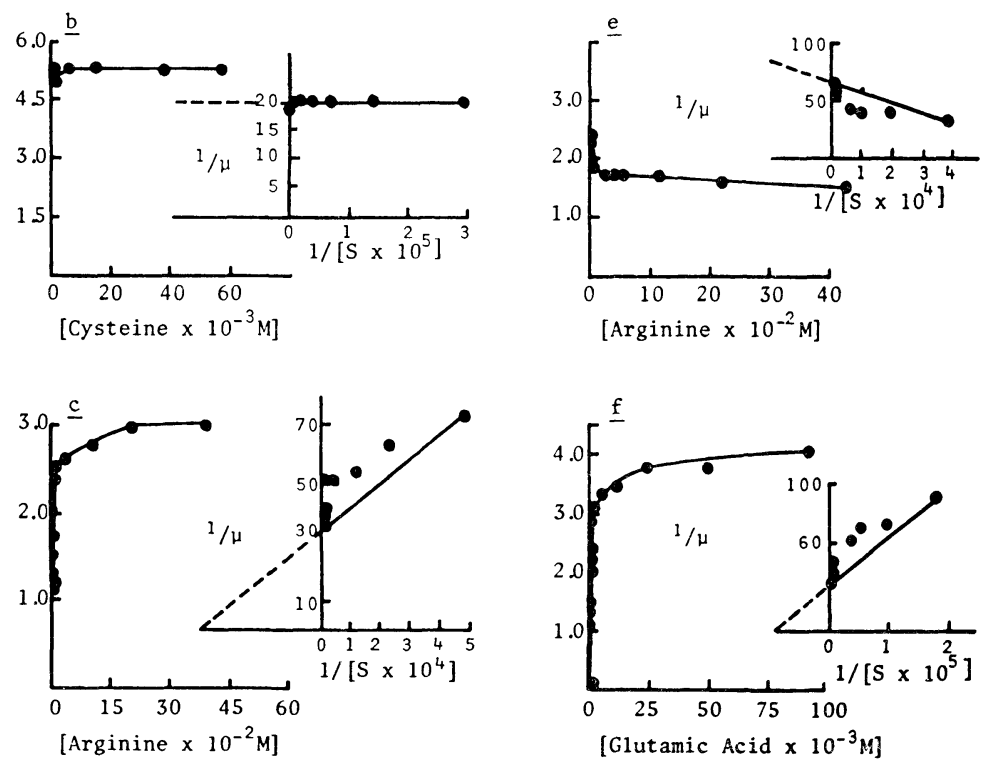

Fig. 1. Growth kinetics studies showing the effect of amino acid concentration on specific growth rate of (a) S. floricola, (b) brevi, (c) S. melliform, (d) S. citri, (e) S. apis and (f) SR-3. Graph insertions of Lineweaver-Burk's reciprocal plots show validity to hyperbolic relations of Monod's growth kinetics of proline (a), arginine (c), leucine (d) and glutamic acid (f). The double reciprocal plots of cysteine (b) and arginine (e) show no relationship to Monod's growth kinetics.

three days for SR-3, four days for $S$. apis and five days for $S$. melliform and $S$. citri. The highest concentration (saturation concentration) or each amino acid except arginine was 32 times that of the concentration of each amino acid in the CDM of CHANG and CHEN (7) and for each amino acid tested one culture contained none of the given amino aeid. The minimum specific growth rate was determined from this culture without the specific amino acid. The highest concentration of arginine was $38 \times 10^{-2} \mathrm{M}$. 
Table 2. The specific growth rate of spiroplasmas grown in medium enriched with and deficient of individual amino acid.

\begin{tabular}{|c|c|c|c|c|c|c|}
\hline \multirow{2}{*}{ Amino acids } & \multicolumn{6}{|c|}{ Specific growth rate $\left(\times 10^{-2} \mathrm{hr}^{-1}\right)$ (maximum/minimum values) } \\
\hline & SR-3 & S. floricola & brevi & S. apis & S. citri & S. melliform \\
\hline L-Alanine & $3.7 / 1.40$ & $4.6 / 3.40$ & $4.8 / 2.80$ & $2.8 / 2.00$ & $2.8 / 1.30$ & $2.7 / 1.40$ \\
\hline L-Arginine & $3.7 / 0.10$ & $4.0 / 2.10$ & - $^{a}$ & - & $2.4 / 1.17$ & $2.9 / 1.40$ \\
\hline \multicolumn{7}{|l|}{ L-Aspartin } \\
\hline acid & $4.2 / 3.50$ & $4.3 / 3.40$ & $4.9 / 3.80$ & $2.8 / 1.40$ & $2.8 / 1.10$ & $2.7 / 0.95$ \\
\hline L-Asparagine & $3.5 / 0.20$ & $4.1 / 0.50$ & $3.9 / 1.30$ & $2.8 / 0.70$ & $2.7 / 0.90$ & $2.7 / 1.30$ \\
\hline L-Cysteine & $4.1 / 3.30$ & $4.6 / 4.00$ & $5.3 / 4.80$ & $2.8 / 2.00$ & $2.8 / 2.20$ & $2.9 / 2.20$ \\
\hline \multicolumn{7}{|l|}{ L-Glutamic } \\
\hline acid & $4.0 / 0.07$ & $4.3 / 1.00$ & $4.0 / 0.90$ & $2.9 / 0.25$ & $2.6 / 0.90$ & $2.7 / 0.92$ \\
\hline L-Gluramine & $4.0 / 0.40$ & $4.4 / 3.50$ & $4.7 / 3.50$ & $2.8 / 2.00$ & $2.6 / 0.40$ & $3.1 / 0.03$ \\
\hline Glycine & $3.9 / 0.10$ & $4.2 / 0.30$ & $4.4 / 1.20$ & $2.7 / 0.60$ & $2.6 / 0.50$ & $2.7 / 0.60$ \\
\hline L-Histidine & $4.0 / 0.40$ & $4.4 / 3.80$ & $5.8 / 4.00$ & $2.9 / 1.40$ & $2.9 / 0.90$ & $2.7 / 1.30$ \\
\hline L-Isoleucine & $3.2 / 0.02$ & $4.0 / 0.04$ & $4.2 / 0.90$ & $2.9 / 1.40$ & $2.7 / 0.40$ & $2.8 / 0.31$ \\
\hline L-Leucine & $3.2 / 0.06$ & $4.2 / 0.70$ & $3.8 / 1.10$ & $2.8 / 0.25$ & $2.7 / 1.08$ & $2.7 / 0.80$ \\
\hline L-Lysine & $3.5 / 0.10$ & $4.1 / 0.40$ & $3.8 / 0.40$ & $2.9 / 0.25$ & $3.0 / 1.10$ & $3.0 / 1.46$ \\
\hline L-Methionine & $3.8 / 2.90$ & $4.3 / 3.60$ & $4.9 / 3.30$ & $2.9 / 2.00$ & $2.9 / 0.13$ & $2.6 / 0.28$ \\
\hline \multicolumn{7}{|l|}{ L-Phenyl- } \\
\hline & $3.6 / 0.50$ & $4.2 / 1.30$ & $4.3 / 1.30$ & $3.0 / 0.90$ & $3.0 / 0.50$ & $2.7 / 1.22$ \\
\hline L-Proline & $3.4 / 0.10$ & $4.4 / 0.24$ & $4.1 / 1.30$ & $2.6 / 0.01$ & $2.7 / 0.20$ & $2.8 / 0.08$ \\
\hline L-Serine & $3.7 / 3.10$ & $4.2 / 3.30$ & $4.9 / 4.20$ & $2.8 / 2.00$ & $2.8 / 2.00$ & $3.0 / 2.30$ \\
\hline L-Threonine & $3.3 / 0.04$ & $4.2 / 0.06$ & $3.6 / 0.40$ & $2.7 / 0.50$ & $2.6 / 0.10$ & $2.7 / 0.31$ \\
\hline L-Tryptophan & $3.9 / 1.80$ & $4.0 / 3.10$ & $5.0 / 4.00$ & $2.8 / 1.20$ & $2.7 / 0.40$ & $2.5 / 0.39$ \\
\hline L-Tyrosine & $3.9 / 2.80$ & $4.6 / 4.10$ & $5.2 / 4.60$ & $2.9 / 2.20$ & $2.7 / 1.40$ & $2.8 / 1.35$ \\
\hline L-Valine & $3.7 / 0.04$ & $4.1 / 0.27$ & $4.4 / 0.60$ & $2.4 / 0.30$ & $2.8 / 1.10$ & $3.1 / 1.10$ \\
\hline
\end{tabular}

$a$ The specific growth rate of spiroplasmas in cultures supplemented with arginine was suppressed compared to cultures without arginine.

\section{RESULTS AND DISCUSSION}

\section{Amino acid deletion}

The effect of amino acid deletion on the growth yield of spiroplasmas is shown in Table 1. An amino acid was considered essential if with deletion the growth yield was depressed to $20 \%$ or less of the yield of the complete control culture (CC). Amino acids are considered to be partially essential if the effect of amino acid deletions on the growth yield varied from 21 to $69 \%$ of the CC yield.

The tests by deletion of individual amino acids from the $\mathrm{CC}$ indicated that of the 20 amino acids tested, 14 were essential for good growth in S. citri, 10 in S. floricola, 10 in S. melliform, 10 in brevi, 7 in S. apis and 14 in SR-3. Asparagine, glutamic acid, glycine, leucine, proline, threonine and valine were essential for growth in all spiroplasmas tested. Deletion of cysteine and serine had marginal growth yield of at least $70 \%$ of that of CC) or no significant effect on growth yield of all spiroplasmas tested. Requirements for other amino acids varied from 
nonessential to essential for good growth. For example, arginine was nonessential for brevi and $S$. apis; partially essential for $S$. melliform, S. floricola, $S$. citri, and essential for SR-3 spiroplasma.

Results of this amino acid deletion study suggest that for optimum growth, spiroplasmas depend on an external supply of most amino acids. All essential and partially essential amino acids determined from the growth study indicated a low biosynthetic capability of spiroplasmas to synthesize most amino acids. The natural habitat of spiroplasma is an environment that contains amino acids such as hemolymph of insects, phloem of plants and nectar of flowers. Mycoplasmas also have been reported to depend on an external supply of amino acids and have low biosynthetic capability $(9,10)$. For example, Mycoplasma mycoides subsp. mycoides in a chemically defined medium (10) was reported to require exogenous supplies of all amino acids, except aspartic acid, cysteine, and glutamic acid.

Results in Table 2 show that the specific growth rate of spiroplasmas was affected by the amino acid composition of the growth medium. Monod (11) showed that the specific growth rate was a function of the substrate concentration that obeys Michaelis-Menten kinetics: $\mu=\mu_{\max }\left[C / K_{s}+C\right]$, where $\mu$ is the specific growth rate at the limiting nutrient concentration $(C), \mu_{\max }$ is the maximum specific growth rate at saturating concentration of the nutrient and $K_{s}$ is the saturation constant, being equal to the substrate concentration supporting the specific growth rate at $0.5 \mu_{\max }$. The ratio of the minimum to the maximum specific growth rate of cultures deleted and enriched respectively with a specific essential or partially essential amino acid at the saturation concentration showed that the growth of spiroplasmas was stimulated by the amino acid (Table 2). For example, the specific growth rate of $S$. floricola in a medium deficient in threonine was $0.06 \times$ $10^{-2} \mathrm{hr}^{-1}$, but the specific growth rate in medium containing $4.8 \times 10^{-2} \mathrm{M}$ amino acid was $4.2 \times 10^{-2} \mathrm{hr}^{-1}$.

In general, the specific growth rate of the other microorganisms is affected by the nutrient composition of the growth medium $(11,12)$. In spiroplasmas, it has been reported that when arginine was added to a chemically undefined medium inoculated with corn stunt spiroplasma, the specific growth rate increased (5).

\section{REFERENCES}

1) A. L. Jones, R. F. Whitcomb, D. L. Williamson, and M. E. Coan, Phytopathology, 67, 738 (1977).

2) E. C. K. Igwegbe, C. Stevens, and J. J. Hollis, Jr., Can. Microbiol., 25, 1125 (1979).

3) R. F. Whiтсомв, Ann. Rev. Microbiol., 34, 677 (1980).

4) I. M. LeE and R. E. Davis, Phytopathology, 74, 84 (1984).

5) C. Stevens, R. M. Cody, and R. T. Gudauskas, J. Gen. Appl. Microbiol., 29, 1 (1983).

6) C. J. Chang and T. A. Chen, J. Bacteriol., 153, 452 (1983).

7) C. J. Chang, T. A. Chen, Science, 215, 1121 (1982). 
8) R. Y. Stanier, E. A. Adelberg, and J. L. Ingraham, In The Microbial World, 4th ed., Prentice-Hall, Englewood Cliffs (1976).

9) S. Razin and A. Cohen, J. Gen. Microbiol., 30, 141 (1963).

10) A. W. Rodwell, J. Gen. Microbiol., 59, 39 (1969).

11) J. Monod, Ann. Rev. Microbiol., 3, 371 (1949).

12) T. E. Shehata and A. G. MarR, J. Bacteriol., 107, 210 (1971). 